\title{
Projective generators and resolutions of identity in Banach spaces
}

\author{
J. ORIHUELA and M. VALDIVIA ${ }^{1}$
}

\begin{abstract}
We introduce the notion of projective generator on a given Banach space. Weakly countably determined and dual spaces with the Radon Nikodym property have projective generators. If a Banach space has projective generator, then it admits a projective resolution of the identity. When a Banach space and its dual both have a projective generator then the space admits a shrinking resolution of the identity. These results include previous ones of Amir and Lindenstrauss, John and Zizler, Gul'ko, Vasak, Tacon, Fabian and Godefroy; and they show how to deal with the general problem of constructing projections and ordering them into a long sequence in a unified way.
\end{abstract}

\section{INTRODUCTION AND NOTATION}

An important and widely open question in the geometry of Banach spaces is that of the existence of non trivial linear continuous operator on a given Banach space. Among such mapping, norm one projections play an important role. In large classes of spaces the projections do exist, and moreover, they can be organized into a "long sequence" with nice properties, something similar to a transfinite Schauder decomposition, called a projectional resolution of identity (P.R.I.). In that way, a powerful tool for studying the structure of the space is provided. Among the applications are: embedding and renorming theorems, results in the theory of Markuševic basis, and properties of compactness in the weak and the $w^{*}$-topologies.

The first non trivial P.R.I. was constructed by Lindenstrauss [L] for reflexive Banach spaces and by Amir and Lindenstrauss [AL] for weakly compactly generated (W.C.G.) spaces. They applied their results to the topological and geometrical structure of weakly compact sets of Banach spaces, now called Eberlein compact spaces. Later Vašák dealt with the P.R.I. for weakly countably determined (W.C.D.) spaces. Independently, Gul'ko, [G], intro-

1 The authors have been partially supported by C.A.I.C.Y.T.

1980 Mathematics Subject Classification (1985 revision): 46B15, 46B20, 46B22

Editorial de la Universidad Complutense. Madrid, 1989. 
duced a topological device to obtain the same results in a very clear and short way, proving at the same time the Corson compactness of the unit ball of the dual of a W.C.D. Banach space, with the weak* topology. The paper of Namioka and Wheeler [NW] gives a precise description of Gul'ko ideas for the weakly compactly generated case. Interesting surveys of these facts can be found in $[\mathrm{P}]$ and $[\mathrm{N}]$. The paper of Talagrand [TA] also studies topological properties, and Orihuela [O] deals with the angelic properties in full generality. Very recently, Mercourakis [M] studied applications of Gul'ko method, Stegall [S2] gave his own version of Vašák results -a relatively simple selfcontained proof- and Valdivia showed how to deal with the construction of a P.R.I. in a very simple way that can be also applied to Fréchet spaces, as well as to more general Banach spaces [Vl-V4].

Whenever we have a projectional resolution of identity in a given Banach space $X$, the adjoint projections give something similar for the weak ${ }^{*}$ topology in $X^{*}$. John and Zizler were the first to study (in a sequence of papers [JZ1-5]) how to deal with the problem of getting a P.R.I. on the dual too, and they applied their methods to obtain renorming theorems as well as shrinking Markuševič basis, see also [S]. The essentially work for W.C.G. Banach spaces with some smoothness property or with a W.C.G. dual space. These results are also treated by Vasák [V] for W.C.D. Banach spaces. Finally, Fabian [F2] has recently dealt with W.C.D. Asplund spaces, and he has shown that they are W.C.G. and admit a Fréchet differentiable norm.

Sometimes it is possible to construct a P.R.I, in the dual $X^{*}$ fo a given Banach space formed by projections which are not necessarily the adjoint of any projection on $X$. The first results in that direction was obtained by Tacon [T] for every very smooth Banach space, and by John and Zizler [JZ5] when $X$ admits a continuously Fréchet differentiable map with bounded non empty support. Both results are included in a general result by Fabian [F1], used as a main tool by Fabian and Godefroy [FG] to prove that every dual Banach space with the Radon Nikodym property admits a P.R.I.

Our aim in this paper is to show how the method to construct projections used by Valdivia in a sequence of papers [V1-V4] can be adapted to describe a unified way of obtaining all the former results. Indeed, we are going to introduce the notion of a projective generator on a given Banach space, and to show that W.C.D. and dual spaces with the Radon Nikodym property have a projective generator. If a Banach space has a projective generator, then it admits a P.R.I. Finally, when for a Banach space $X$, and its dual $X^{*}$ both have a projective generator, then a P.R.I. exists on $X$ such that the adjoint maps are a P.R.I. on $X^{*}$.

The key for our exposition lies in the notion of norming pair. In Section 
2 we describe their basic properties and relate this notion with the one used by Gul'ko [G]. In Section 3 we see how to get norm one projections from norming pairs and the notion of projective generator comes naturally. In Section 4 we describe the P.R.I. associated with a projective generator and in the last section we see when it can be constructed in such a way that the adjoints are a P.R.I. on the dual space.

We are grateful to G. Godefroy and J.E. Jayne for converstions on some aspects of the content of this paper.

We have been using the classical notation which can be found for instance in [D1-D2]. The closed unit ball of a Banach space $X$ is denoted by $B(X)$. The vector spaces we shall use here are defined over the field $\mathrm{K}$ of real or complex numbers. If $\mathrm{K}$ is the real field, $H$ denotes the field of the rational numbers, if $\mathrm{K}$ is the complex field, $H$ denotes the field of numbers $\{a+i b: a, b$ are rationals . Given a Banach space $X$ and $x$ in $X$, we write $\hat{x}$ to denote the same element as a continuous function on $X^{*}\left[\sigma\left(X^{*}, X\right)\right]$. The density character of a topological space is the smallest cardinality of a dense subset. For a Banach space $X$ we write $X_{\sigma}^{*}$ to denote the weak* dual $X^{*}\left[\sigma\left(X^{*}, X\right)\right]$.

\section{NORMING PAIRS}

Let $X$ be a Banach space. If $A$ is a subset of $X$ and $B$ a subset of $X^{*}$, the pair $(A, B)$ is called a preconjugate pair if

(i) $\left\{\hat{a}_{\mid} B^{: a \in A}\right\}$ is pointwise dense in $\left\{\hat{x}_{B}: x \in X\right\}$

and

(ii) $\left\{\hat{b}_{\mid}: b \in B\right\}$ is pointwise dense in $\left\{\hat{y} A^{\left.: y \in X^{*}\right\}}\right.$

This notion was introduced by S. Gul'ko in the setting of paired topological spaces to deal with a general version of the theorems of Amir and Lindenstrauss, $[A L],[G],[N]$. Among other interesting results, Gul'ko constructs non trivial preconjugate pairs in any given Banach space $X$. We are interested here in a particular kind of preconjugate pair:

Definition 1. A preconjugate pair $(A, B)$ in the Banach space $X$ is called a norming pair if $A$ and $B$ are $H$-linear subspaces that verify:

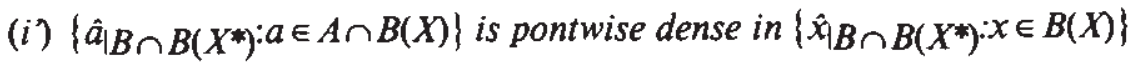

(ii) $\left\{\hat{b}_{L A \cap B(X)}: b \in B \cap B\left(X^{*}\right)\right\}$ is pointwise dense in $\left\{\hat{y} A \cap B(X)^{\left.: y \in B\left(X^{*}\right)\right\}}\right.$ 
Using the terminology of Gul'ko we could say that a preconjugate pair of H-linear subspaces $(A, B)$ is a norming pair when the pair

$$
\left(A \cap B(X), B \cap B\left(X^{*}\right)\right)
$$

is also a preconjugate pair of the pairing formed by $B(X)$ and $B\left(X^{*}\right)$ endowed with the weak and the weak* topology, respectively, with the duality mapping $<x_{3} f>=f(x), x$ in $X$ and $f$ in $X^{*},[\mathrm{G}]$.

Our motivation for this name is made clear in the following result. It shows that a norming pair is nothing other than a pair of $\mathrm{H}$-linear subspaces $A$ of $X$ and $B$ of $X^{*}$ such that $A$ is a norming set for $B$, and $B$ is a norming set for $A$. Indeed, in what follows we could think of this property as the definition of a norming pair and we could go straight to proposition 2. Nevertheless, we prefer to give proposition 1 to show the link between Gul'ko ideas and our constructions. In that way a question of M. Fabian is answered [F1, pp. 366].

Proposition 1.Let $X$ be a Banach space, and let $A \subset X$ and $B \subset X^{*}$ be H-linear subspaces. The following conditions are equivalent:

(i) $(A, B)$ is a norming pair,

(ii) The following two conditions hold

ii ) If $x \in A$, then $\|x\|=\sup \left\{\left|<x_{1} f>\right| f \in B\left(X^{*}\right) \cap B\right\}$

ii ) If $f \in B$, then $\|f\|=\sup \{|<x, f>|: x \in B(X) \cap A\}$

(iii) If $\bar{A}$ and $\bar{B}$ are the closures of $A$ and $B$ in the norm topologies, then they also verify conditions $\left(i i_{1}\right)$ and $\left(i i_{2}\right)$ above.

Proof. $i) \Rightarrow$ ii) Let $x$ be an element of $A \cap B(X)$. The density condition (ii) tells us that $\left(i i_{1}\right)$ is verified for $\|x\|$. In case $\|x\|>1$, and $x$ in $A$, we take a rational number $r>0$ with $\|r x\| \leq 1$, then $r x$ belongs to $A \cap B(X)$ and $\left(i i_{1}\right)$ is verified for $\|r x\|$. It follows that $\|x\|$ also verifies it. The same argument proves $\left(i i_{2}\right)$ using $\left(i^{\prime}\right)$ instead of $(i i)$.

ii) $\Rightarrow$ iii) Let $x$ be an element of $\bar{A}$ and $\varepsilon>0$. There is some $a$ in $A$ with $\|x-a\| \leq \varepsilon$. We have

$$
\begin{aligned}
& \|x\| \leq\|x-a\|+\|a\| \leq \varepsilon+\sup \left\{|<a, f>| f \in B\left(X^{*}\right) \cap B\right\} \leq \\
& \leq \varepsilon+\sup \left\{\mid<a-x_{*} f>1: f \in B\left(X^{*}\right) \cap B\right\}+ \\
& +\sup \left\{\mid<x, f>1 \cdot f \in B\left(X^{*}\right) \cap B\right\} \leq \\
& \leq \varepsilon+\|x-a\|+\sup \left\{\mid<x, f>1: f \in B\left(X^{*}\right) \cap B\right\} \\
& \leq 2 \varepsilon+\sup \left\{\mid<x, f>i f \in B\left(X^{*}\right) \cap B\right\} \leq 2 \varepsilon+\|x\|
\end{aligned}
$$

from which $i i_{1}$ ) follows letting $\varepsilon$ tend to zero. 
The same argument proves $i i_{2}$ ) for the closure of $B$.

iii) $\Rightarrow i$ ) Let $R_{A}$ be the restriction mapping from $X^{*}$ onto $\bar{A}^{*}$. Condition $i i_{2}$ ) says that $R_{A}$ gives an isometry from $\bar{B}$ in to $\bar{A}^{*}$, and condition $i i_{1}$ ) together with the Hahn-Banach theorem says that $R_{A}(\bar{B})$ is $\sigma\left(\bar{A}^{*}, \bar{A}\right)$-dense in $\bar{A}^{*}$. Therefore, $R_{A}(B)$ is $\sigma\left(\bar{A}^{*}, A\right)$-dense in $\bar{A}^{*}$. Analogously, we obtain for the restriction mapping $R_{B}$ from $X^{* *}$ onto $\bar{B}^{*}$ that $R_{B}(A)$ is $\sigma\left(\bar{B}^{*}, B\right)$-dense in $\bar{B}^{*}$. In other words, we have proved that $(A, B)$ is a preconjugate pair in $X$. To finish the proof we need to show that the conditions of definition 1 are also verified. We see that $R_{A}\left(\bar{B} \cap B\left(X^{*}\right)\right)$ is $\sigma\left(\bar{A}^{*}, \bar{A}\right)$-dense in $B\left(\bar{A}^{*}\right)=R_{A}\left(B\left(X^{*}\right)\right)$ because of condition $\left.i i_{1}\right)$ and the Hahn-Banach separation theorem. It follows that $R_{A}\left(B \cap B\left(X^{*}\right)\right)$ is $\sigma\left(\bar{A}^{*}, A\right)$-dense in $R_{A}\left(B\left(X^{*}\right)\right.$ ). Dealing with $\left.i i_{2}\right)$ we obtain, in the same way that $R_{B}(A \cap B(X))$ is $\sigma\left(B^{*}, B\right)$-dense in $R_{B}(B(X))$. Indeed, we have proved that $\left(A \cap B(X), B \cap B\left(X^{*}\right)\right)$ is a preconjugate pair in $\left(B(X), B\left(X^{*}\right)\right)$.

Q.E.D.

Corollary 1.1. If $(A, B)$ is a norming pair in the Banach space $X$, then the norm closures $(\bar{A}, \bar{B})$ form a norming pair of norm-closed subspaces of $X$ and $X^{*}$, respectively.

The next proposition shows how to build up non-trivial norming pairs in a given Banach space $X$. The general construction of Gul'ko in [G,lemma 3] could be applied here with some minor modifications. Nevertheless, we shall follow an argument of Valdivia, [V1], based on an idea of Mazur, to describe this construction in the richer structure provided by the Banach space.

Proposition 2. Let $X$ be a Banach space and $(G, W)$ a norming pair in $X$. Let $A_{0} \subset G$ and $B_{0} \subset W$ be two infinite subsets with $\left|A_{0}\right| \leq \lambda$ and $\left|B_{0}\right| \leq \lambda$. There is a norming pair $(A, B)$ in $X$ with

$$
A_{0} \subset A \subset G, \quad B_{0} \subset B \subset W,|A| \leq \lambda,|B| \leq \lambda .
$$

Proof. For every $x \in G$ let us denote by $\psi(x)$ a countable subset of $W$ such that

$$
\|x\|=\sup \{1<x, f>\mid \cdot f \in \psi(x)\} .
$$

Analogously, for every $f \in W$ denote by $\varphi(f)$ a countable subset of $G$ such that

$$
\|f\|=\sup \{\mid<x, f>1: x \in \varphi(f)\} .
$$

Now we are going to proceed by recurrence, let us suppose that for a given non negative integer $p$, and every non negative integer $m \leq p$ we have obtained subsets

$$
A_{m} \subset G, B_{m} \subset W,\left|A_{m}\right| \leq \lambda,\left|B_{m}\right| \leq \lambda
$$


We write $C_{m}$ and $D_{m}$ for the $\mathrm{H}$-linear hulls of $A_{m}$ and $B_{m}$, respectively. We define

$$
B_{p+1}:=D_{p} \cup\left\{\psi(x): x \in C_{p}\right\}, A_{p+1}:=C_{p} \cup\left\{\varphi(f): f \in D_{p}\right\} .
$$

If we take

$$
A=\cup\left\{A_{n}: n=0,1,2, \ldots\right\} \text { and } B=\cup\left\{B_{n}: n=0,1,2, \ldots\right\}
$$

then $(A, B)$ is a norming pair in $X$ with

$$
A_{0} \subset A \subset G, B_{0} \subset B \subset W \text { and }|A| \leq \lambda,|B| \leq \lambda .
$$

Proposition 3. Let $(A, B)$ be a norming pair in the Banach space $X$. Then

a) If $x \in A$ and $y \in B^{\perp}$, then $\|x\| \leq\|x+y\|$.

b) If $f \in A^{\perp}$ and $g \in B$, then $\|g\| \leq\|g+f\|$.

Proof. a) $\|x\|=\sup \left\{|<x,>| f \in B\left(X^{*}\right) \cap B\right\}=$

$$
\begin{aligned}
& =\sup \left\{\mid<x+y, f>l: f \in B\left(X^{*}\right) \cap B\right\} \leq \\
& \leq \sup \left\{\mid<x+y, f>l: f \in B\left(X^{*}\right)\right\}=\|x+y\|
\end{aligned}
$$

b) The same argument works in this case.

Q.E.D.

We can summarize in the following:

Proposition 4. Let $X$ be a Banach space, and $A_{0} \subset X$ and $B_{0} \subset X^{*}$ infinite subsets with $\left|A_{0}\right| \leq \lambda$ and $\left|B_{0}\right| \leq \lambda$. Then there exist norm closed subspaces $E$ of $X$ and $F$ of $X^{*}$ such that
a) If $x \in E,\|x\|=\sup \{\mid<x, f>1: f \in B(F)\}$
b) If $f \in F,\|f\|=\sup \{|<x, f>|: x \in B(E)\}$
c) If $x \in E$ and $y \in F^{\downarrow}$, then $\|x\| \leq\|x+y\|$
d) If $f \in E^{1}$ and $g \in F$, then $\|g\| \leq\|g+f\|$
e) $E \supset A_{0}$ dens $E \leq \lambda ; F \supset B_{0}$, dens $F \leq \lambda$.

Proof. $E$ and $F$ are the norm closure of the norming pair constructed in Proposition 2.

Q.E.D.

\section{FROM NORMING PAIRS TO LINEAR PROJECTIONS}

We now describe when a given norming pair is already a conjugate pair and how it gives a norm-one projection. 
Propositon 5. Let $X$ be a Banach space and $(E, F)$ a norming pair of norm closed subspaces of $X$ and $X^{*}$, respectively. The following conditions are equivalent:

i) There is a norm one projection of $X$ onto $E$ along $F^{\perp}$.

ii) $X$ is the norm closure of $E+F^{1}$.

iii) $E^{\perp} \cap F^{6\left(X^{*}: x\right)}=\{0\}$.

iv) The restriction mapping $R_{F}$ from $X$ onto the dual of $F\left[\sigma\left(X^{*}, X\right)\right]$ applies $E$ onto that dual space.

Proof. i) $\Rightarrow$ ii) This is obvious.

ii) $\Leftrightarrow$ iii) By polarity

i) $\Rightarrow$ iv) Let $f$ be any element of the dual of $F\left[\sigma\left(X^{*}, X\right)\right]$. By the HahnBanach theorem there is some $g \in X$ such that $g_{v}=f$. If $g=g_{1}+g_{2}$, with $g_{1}$ in $E$ and $g_{2}$ in $F^{1}$, then $g_{\mid F}=g_{\text {if }}=f$ from where the conclusion follows.

iv) $\Rightarrow$ i) Let $\xi$ be the restriction mapping from $E$ onto the dual of $F\left[\sigma\left(X^{*}, X\right)\right]$, $\xi(x):=\hat{x}_{F F}$, iv) means that $\xi$ is a bijection and therefore we can define

$$
P(x):=\xi^{-1}\left(\hat{x}_{v}\right) \text { for every } x \text { in } X
$$

It is clear that $P$ is a norm one projection of $X$ onto $E$

Q.E.D.

Definition 2. A norming pair satisfying the equivalent conditions of Proposition 5 is called a conjugate pair.

Indeed, it is not difficult to show that our definition 2 is almost a particular case of the one given by Gul'ko,[G],in a topological setting.

Let us now show how to apply the former proposition in a concrete example:

Corollary 5.1. Let $X$ be a reflexive Banach space. Then every norming pair of norm closed subspaces $(E, F)$ of $X$ and $X^{*}$, respectively, is already a conjugate pair giving a norm one projection of $X$ onto $E$ along $F^{\perp}$.

Proof. In this case we have

$$
E^{\perp} \cap \bar{F}^{(x \cdot x)}=E^{\perp} \cap \bar{F}^{\| I !}=E^{\perp} \cap F=\{0\},
$$

if we bear in mind condition (b) of Propositon 3 for the norming pair $(E, F)$.

Q.E.D.

So, in order to obtain Lindenstrauss theorem, [L], it is enough to apply Proposition 4 together with Corollary 5.1: 
Corollary 5.2,[L]: If $X$ is a reflexive Banach space and $N$ is a closed subspace of $X$, there is a closed subspace $E$ containing $N$ with dens $E=$ dens $N$ and $a$ norm one projection from $X$ onto $E$.

In more general cases this result is not true. In order to be able to deal with this problem it is necessary to involve more structure of $X$ in the construction of the norming pair. To be more precise we shall need the following definition in an arbitrary Banach space $X$.

Definition 3. Let $W$ be a norming subset of $X^{*}$ and $\varphi$ a set valued map from $W$ into $2^{x}$ verifying the following conditions:

(3.1) $\varphi(f)$ is a countable subset of $X$ for every $f \in W$.

(3.2) $\|f\|=\sup \{|<x, f>|: x \in \varphi(f)\}$.

(3.3) For every norming pair $(E, F)$ in $X$ such that $F \subset W$ and $\varphi(F) \subset E$ the pair of norm closures $(\bar{E}, \bar{F})$ is a conjugate pair in $X$.

Then $\varphi$ is called a projective generator on the Banach space $X$.

If $(E, F)$ is a norming pair that verifies (3.3) above we say that the norm one projection of $X$ onto the closure of $E$ along $F^{\perp}$ is based on $(E, F)$.

We have the following:

Theorem 1. Let $X$ be a Banach space with a projective generator $\varphi$ defined on a norming $H$-subspace $W$ of $X^{*}$. Let $A_{0}$ and $B_{0}$ be infinite subset of $X$ and $W$, respectively, with

$$
\left|A_{0}\right| \leq \lambda \quad \text { and } \quad\left|B_{0}\right| \leq \lambda
$$

Then there is a norm one projection $P$ of $X$, based on a norming pair $(A, B)$ with

$$
A_{0} \subset A, B_{0} \subset B,|A| \leq \lambda \text { and }|B| \leq \lambda
$$

and, consequently,

$$
A_{0} \subset P(X), \text { dens } P(X) \leq \lambda, B_{0} \subset P^{*}\left(X^{*}\right), \text { dens } P^{*}\left(X^{*}\right)_{\sigma} \leq \lambda
$$

Proof. $(X, W)$ is a norming pair and we can construct a norming pair $(A, B)$ in $X$ such that:

$$
A_{0} \subset A \subset X, B_{0} \subset B \subset W,|A| \leq \lambda \text { and }|B| \leq \lambda
$$

and $\varphi(B) \subset A$ as we have done in Proposition 2. Let us remark that we are working here with our particular generator $\varphi$ instead of an arbitrary norming mapping. Condition (3.3) says that the projection based on $(A, B)$ has the required properties.

Q.E.D. 
Example. In every weakly countably determined Banach space $X$ there is a projective generator defined on all of $X^{*}$.

Proof. There is a sequence $\left(B_{n}\right)$ of closed unit balls around the origin that determine $X$ in $X^{* *}\left[\sigma\left(X^{* *}, X^{*}\right)\right],[\mathrm{V}],[\mathrm{V} 3]$. That means

$$
X=\bigcup\left\{\bigcap_{j=1}^{\infty} \bar{B}_{a_{j}}^{\alpha x^{w} \times x^{n}}:\left(a_{j}\right) \in \mathbb{N}^{N}\right\}
$$

where some of the intersections used could be void. For every $f$ in $X^{*}$ we take $\varphi(f)$ verifying conditions (3.1), (3.2) and such that

$$
\sup \left\{1<x, f>1: x \in B_{n}\right\}=\sup \left\{\mid<x, f>1: x \in B_{n} \cap \varphi(f)\right\} n=1,2, \ldots
$$

Let us show now that $\varphi$ is a projective generator; let $(E, F)$ be a norming pair with $\varphi(F) \subset E$. We are going to show that $\mathrm{E}^{\perp} \cap \bar{F}^{\alpha\left(x^{2},\right)^{\prime}}=\{0\}$ and so $(\bar{E}, \bar{F})$ verifies the equivalents conditions of Proposition 5 and is a conjugate pair. Let $f$ be an element of $E^{\perp} \cap \bar{F}^{\propto \alpha \cdot x)}$ and suppose $f \neq 0$. There is some $x_{0} \in X$ with $\left|f\left(x_{0}\right)\right|=1$, and a net $\left\{f_{f} j \in D, \geq\right\}$ in $F$ that converges to $f$ for the Mackey topology $\mu\left(X^{*}, X\right)$. There is a sequence $n_{1}<n_{2}<\ldots<n_{j}<\ldots$

of positive integers such that if $A=\bigcap_{j=1}^{\infty} \bar{B}_{n_{j}}^{\alpha x+x q}$ we have $x_{0} \in A \subset X$.

Let $0<\varepsilon<1 / 2$ and $j_{0} \in D$ such that

$$
\sup \left\{\left|f_{0}(x)-f(x)\right|: x \in A\right\}<\varepsilon .
$$

There is some positive integer $h$ such that

$$
\sup \left\{\left|f_{j_{0}}(x)-f(x)\right|: x \in B_{n_{h}}\right\}<\varepsilon
$$

and, in particularly, $\left|f_{j_{0}}\left(x_{0}\right)-f\left(x_{0}\right)\right|<\varepsilon$ from which it follows that $\left|f_{j_{0}}\left(x_{0}\right)\right|>1-\varepsilon$.

But if we look at the definition of $\varphi$ we have some $y_{0} \in B_{n_{h}} \cap \varphi\left(f_{j_{0}}\right)$ such that $\left|f_{J_{0}}\left(y_{0}\right)\right|>I-\varepsilon$.

Therefore

$$
\left|f\left(y_{0}\right)\right| \geq\left|f_{j_{0}}\left(y_{0}\right)\right|-\left|f\left(y_{0}\right)-f_{j_{0}}\left(y_{0}\right)\right|>1-\varepsilon-\varepsilon=1-2 \varepsilon>0 .
$$

So $f\left(y_{0}\right) \neq 0$ with $y_{0} \in \varphi(F) \subset E$, which is a contradiction

Q.E.D.

If we have a norm-one projection $P$ on $X$ and $P(X)=E$, then the adjoint mapping $P^{*}$ is a norm-one projection and the restriction mapping $R_{E}$ from $X^{*}$ onto $E^{*}$ is an isometry from $P^{*}\left(X^{*}\right)$ onto $E^{*}$. Nevertheless, it could be that the 
conditions of Proposition 5 are not satisfied but we still have a norm one Projection on the dual $X^{*}$. In such a case the projection is of course not weak* continuous. The analogous result to Propostion 5 for the dual says as follows:

Proposition 6. Let $X$ be a Banach space and $(E, F)$ a norming pair of norm closed subspaces of $X$ and $X^{*}$, repectively. The following conditions are equivalent:

(i) There is a norm one projection of $X^{*}$ onto $F$ along $E^{\perp}$

(ii) $X^{*}$ is the norm closure of $F+E^{1}$

(iii) $F^{\perp} \cap \bar{E}^{\alpha(X-x)}=\{0\}$

(iv) The restriction mapping $R_{E}$ from $X^{*}$ onto $E^{*}$ maps $F$ onto $E^{*}$.

Proof. It is enough to apply Proposition 5 to the norming preconjugate pair $(F, E)$ in $\left(X^{*}, X^{* *}\right)$.

Q.E.D.

It seems to be natural to ask for projective generator in $X^{*}$ defined on $X$. We rewrite again our Definition 3 in that case:

Definition 4. Let $\Psi$ be a set-valued map from $X$ into $2^{x^{*}}$ verifying:

(5.1) $\psi(x)$ is countable for every $x$ in $X$.

(5.2) $\|x\|=\sup \left\{\left|<x, y^{*}>\right|: y^{*} \in \psi(x)\right\}$.

(5.3) For every norming preconjugate pair $(E, F)$ in $X$ such that $\psi(E) \subset F$, then the pair of norm closure $(\bar{E}, \bar{F})$ verifies the equivalent conditions of Proposition 6.

Then $\psi$ is called a projective generator on $X^{*}$.

We can read our theorem 1 for this case:

Teorem 2. Let $X$ be a Banach space with a projective generator on $X^{*}$. Let $A_{0} \subset X$ and $B_{0} \subset X^{*}$ be infinite subset with

$$
\left|A_{0}\right| \leq \lambda \text { and }\left|B_{0}\right| \leq \lambda
$$

Then there is a norm-one projection $P$ of $X^{*}$ such that

$$
P\left(X^{*}\right) \supset B_{0}, \text { dens } P\left(X^{*}\right) \leq \lambda, P^{*}\left(X^{* *}\right) \supset A_{0} \text { dens } P^{*}\left(X^{* *}\right)_{\sigma} \leq \lambda .
$$

\section{Examples:}

(A) Let $X$ be a Banach space with a Gâteaux-differentiable norm \|. $\|$ with norm-weak derivative:

$$
X \backslash\{\} \rightarrow X^{*} \backslash\{\mid 0\}
$$


where $f_{x}$ is the only vector in the unit sphere of $X^{*}$ with $\left\langle x_{1} f_{x}\right\rangle=\|x\|$

If we define $\varphi(x)=\left\{f_{x}\right\}$ and $\varphi(0)=\{0\}$, then the mapping $\varphi$ is a projective generator on $X^{*}$.

Proof. Take a norming pair $(E, F)$ in $X$ and suppose that $\varphi(E) \subset F$. The normweak continuity of the derivative implies that $\varphi(\bar{E}) \subset \bar{F}$. Now, the BishopPhelps theorem says that the restriction mapping $R_{E}$ from $X^{*}$ onto $\bar{E}^{*}$ is an onto mapping restricted to $\bar{F}$, so condition (iv) of Propositon 6 holds and the proof is finished.

Q.E.D.

The construction of projections in duals of Banach spaces with Gâteauxdifferentiable norm having norm-weak derivative was done by Tacon, [T]. Extensions of his results was obtained by John and Zizler, [JZ4], Recently M. Fabian has obtained a more general version of these results [F1] that we include in the following example:

(B) Let $X$ be a Banach space admitting a norm-weak continuous mapping $D$ defined on an open subset $B$ of $X$ onto $X^{*}$ such that

$$
\overline{s p}\left\{D x_{1}: x \in V \cap B\right\}=V^{*}
$$

for every subspace $V$ of $X$. Then $X$ admits a projective generator on $X^{*}$.

Proof. Indeed, for every $x$ in $X$, take $v_{x} \in B_{X^{*}}$ with $\left\langle x, v_{x}\right\rangle=\|x\|$, and we define

$$
\varphi(x)= \begin{cases}\left\{v_{x} D x\right\} & \text { if } x \in B \\ \left\{v_{x}\right\} & \text { otherwhise }\end{cases}
$$

Then $\varphi$ is a projective generator on $X^{*}$. Take a norming pair $(E, F)$ in $X$ and suppose that $\varphi(E) \subset F$.

The norm-weak continuity of the mapping $D$ ensures that

$$
D(\bar{E} \cap B) \subset \bar{F},
$$

from which it follows that the restriction mapping $R_{\varepsilon}$ from $X^{*}$ onto $\bar{E}^{*}$ is an onto map when restricted to $\bar{F}$. Thus condition (iv) of Proposition 6 holds and the proof is complete.

Q.E.D.

Indeed, we could say that the first projective generator on $X^{*}$ was implicitely used by Fabian, [F1], in the way shown above. Nevertheless, he did not 
know if Gul'ko's approach could be adapted to obtain his result. By our construction it seems to be clear how to do it.

The results of [F1] have beem used by Fabian and Godefroy in [FG] to obtain projections in the dual of Asplund spaces. We shall see now that Asplund spaces are exactly the Banach spaces admitting a projective generator on $X^{*}$.

Recall the following result, which has its origin in the work of R.C. James, [see 61-62].

If $X$ is a real Banach space with separable dual and $C$ is a closed convex subset of $B\left(X^{*}\right)$ such that for every $x$ in $X$ there is a point $f_{x}$ in $C$ with $\left\langle x, f_{x}\right\rangle=\|x\|$, then $C$ is a weak ${ }^{*}$ compact subset of $X^{*}$.

It is clear that for a complex Banach space the same is true, it is enough to have a look on the real underlying structure.

Theorem 3. Let $X$ be a Banach space. $X$ is an Asplund space if and only if it has a projective generator on $X^{*}$.

Proof. First suppose that $X$ admits a projective generator $\psi$ on $X^{*}$. Let $N$ be a separable subspace of $X$. By Theorem 2 we know that there is a separable subspace $E$ of $X$ and norm one projection $P$ of $X^{*}$ such that $E \supset N$ and $P\left(X^{*}\right)$ is separable and isometric with $E^{*}$ through the restriction map $R_{E}$. So $E^{*}$ will also be separable and the dual $N^{*}$ will also be separable too. Consequently, the space $X$ is an Asplund space, bearing in mind the result of Stegall [S1]

Reciprocally, if $X$ an Asplund space and we consider the \|\| -weak* usco map defined by

$$
\begin{gathered}
\phi: X \rightarrow 2^{\text {Axक }} \\
\phi(x)=\left\{u \in B\left(X^{*}\right):<x, u>=\|x\|\right\}
\end{gathered}
$$

If apply the selection theorem of Jayne and Rogers [JR], then $\phi$ has a firstBaire class selector $f$ for the norm topologies, we mean $f: X \rightarrow B\left(X^{*}\right)$ is such that $f(x) \in \phi(x)$, for every $x$ in $X$, and there is a sequence of $\|f\|-\|\|$ continuous maps

$$
f_{\pi}: X \rightarrow B\left(X^{*}\right)
$$

with

$$
\|\|-\lim _{n \rightarrow \infty} f_{n}(x)=f(x)
$$


for every $x$ in $X$.

If we denote by

$$
\psi: X \rightarrow 2^{x^{*}}
$$

the map

$$
\psi(x)=\left\{f(x), f_{1}(x), f_{2}(x), \ldots f_{n}(x), \ldots\right\}
$$

we obtain a projective generator on $X^{*}$. Indeed, first consider a norming preconjugate pair $(E, F)$ in $X$ with $\psi(E) \subset F$ and the closures $\bar{E}$ and $\bar{F}$ separable subspaces of $X$ and $X^{*}$, respectively. It is clear that $\psi(\bar{E}) \subset \bar{F}$, therefore $R_{E}(B(\bar{F})$ ) verifies the conditions of Godefroy's, theorem so it is a weak* compact subset of $B\left(\bar{E}^{*}\right)$. Thus it coincides with $B\left(\bar{E}^{*}\right)$ because of the weak* density condition we have for a norming pair. The general case easily follows from a reduction argument to the separable case. Indeed, let $(E, F)$ be a norming pair in $X$ formed with non separable subspaces, let us take $g$ in $\bar{E}^{*}$. Denote by $G$ the subspace $R_{E}(\bar{F})$, by $A_{0}$ a countable subset of $B(\bar{E})$ that norms $g$, and by $B_{0}$ the countable subset of $\bar{E}^{*}$ given by $R_{E}\left(\psi\left(A_{0}\right)\right)$, which is contained in $G$ because $\psi(\bar{E}) \subset \bar{F}$. We can now construct a norming pair $(A, B)$ in $\bar{E}$ such that $A$ and $B$ are countable,

$$
R_{f}(\psi(A)) \subset B \subset G,
$$

and $A$ is a norming set of the linear maps in the set

$$
\{g-b: b \in B\} \subset \bar{E}^{*}
$$

Therefore, $\left(A,\left(R_{\varepsilon}\right)^{-1}(B)\right)$ is a norming pair in $X$ of separable subspaces with $\psi(A) \subset\left(R_{E}\right)^{-1}(B)$. As the separable case has been proved above, there is some $s$ belonging to the norm closure of $\left(R_{\varepsilon}\right)^{-1}(B)$ such that $s$ coincides with $g$ on $\bar{A}$. Moreover, for some sequence $\left(s_{n}\right)$ in $\left(R_{E}\right)^{-1}(B)$ we have that $\left(s_{n}\right)$ converges to $s$ in $X^{*}$. By the condition imposed on $A$ is clear that $\left(s_{n}\right)$ converges to $g$ in $E^{*}$. Therefore we have $g=R_{E}(s)$ and the proof is finished.

Q.E.D.

Note that Fabian and Godefroy, $[\mathrm{FG}]$, show how the selector constructed above gives a map $D$ verifying the conditions of Example (B) above. Our argument only differs in the way used to reduce to the separable case.

Finally, we note what happens if we have a projective generator on an Asplund space $X$ too: 
Theorem 4. Let $X$ be an Asplund space with a projective generator $\varphi$ on $X$ defined on $X^{*}$. Let $A_{0} \subset X$ and $B_{0} \subset X$ be infinite subsets with

$$
\left|A_{\mathrm{o}}\right| \leq \lambda \text { and }\left|B_{\mathrm{o}}\right| \leq \lambda
$$

Then there is a norm one projection $P$ in $X$ based on a norming pair $(A, B)$ with

$$
A_{0} \subset A, B_{0} \subset B,|A| \leq \lambda,|B| \leq \lambda
$$

and such that $P^{*}$ is based on the norming pair $(B, A)$ of $X^{*}$.

Consequently

$$
A_{0} \subset P(X), \mathrm{B}_{0} \subset P^{*}\left(X^{*}\right)=\bar{B}^{n ! n}, \operatorname{dens} P(X) \leq \lambda, \operatorname{dens} P^{\star}\left(X^{*}\right) \leq \lambda .
$$

Proof. Let $\psi$ be the projective generator of $X^{*}$ constructed above. We can construct the norming pair $(A, B)$ in $X$ such that

$$
A_{0} \subset A \subset X, B_{0} \subset B \subset X^{*},|A| \leq \lambda,|B| \leq \lambda
$$

and

$$
\varphi(B) \subset A, \psi(A) \subset B
$$

as we have done in Proposition 2. Note that we are working here with our particular generators $\varphi$ and $\psi$ instead of and arbitrary norming mapping. It is quite clear that $P^{*}$ is nothing other than the projection of $X^{*}$ onto $\bar{B}^{\text {\#ll }}$.

Q.E.D.

\section{PROJECTIONAL RESOLUTIONS OF IDENTITY}

We have seen how to construct projections on a given Banach space with a projective generator. In this section we shall see that they can be organized into a "long sequence" with nice properties. In order to be more precise we start with a classical definition:

Definition 5. Let $X$ be a Banach space. A projective resolution of identity (P.R.I) is a well ordered family

$$
\left\{P_{\mathrm{a}}: \omega_{\mathrm{o}} \leq \alpha \leq \mu\right\}
$$

of projections, where $\mu$ is the first ordinal with $|\mu|=$ dens $X$, which satisfies the following conditions: 
(i) $\left\|P_{\mathrm{a}}\right\|=1$ for every $\alpha$

(ii) dens $\left(P_{0}(X)\right) \leq|\boldsymbol{\alpha}|$ for every $\alpha$

(iii) $P_{\mathrm{a}} P_{\mathrm{\beta}}=P_{\beta} P_{\mathrm{a}}=P_{\beta}$ if $\omega_{s} \leq \beta \leq \alpha \leq \mu$

(iv) ${\underset{\beta<a}{\cup} P_{\beta+1}(X)}^{1: 1}=P_{a}(X)$ for every $a$

(v) $P_{\mathrm{p}}=I_{x}$

In the above statement, lal denotes the cardinality of an ordinal $\alpha$. We immediately have the following.

Therorem 4. Let $X$ be a Banach space with a projective generator $\varphi$ based on a norming subspace $W$ of $X^{*}$. Then $X$ admits a projectional resolution of the identity $\left\{P_{a} \cdot \omega_{a} \leq \alpha \leq \mu\right\}$ where every $P_{a}$ is based on a norming pair $\left\{A_{a}, B_{a}\right\}$ such that

and

$\left|A_{\alpha}\right| \leq \alpha,\left|B_{\alpha}\right| \leq \alpha$ for every $\alpha$,

$A_{\beta} \subset A_{\mathrm{a}}$ and $B_{\beta} \subset B_{\mathrm{a}}$ if $\omega_{\mathrm{o}} \leq \beta \leq \alpha \leq \mu$.

$\cup\left\{A_{\beta}: \omega_{o} \leq \beta<\alpha\right\}$ is equal to $A_{a}$ for every ordinal limit $\alpha$,

$\cup\left\{B_{\beta}: \omega_{o} \leq \beta<\alpha\right\}$ is equal to $B_{\mathrm{a}}$ for every ordinal limit $\alpha$.

Proof. Take a dense subset $\left\{x_{a}: \alpha<\mu\right\}$ of $X$, where $\mu$ is the first ordinal with $|\mu|=$ dens $X$.

Let $A_{0}$ be equal to $\left\{x_{\alpha}: \alpha<\omega_{0}\right\}$, and $B_{0}$ be a countable subset of $W$ norming every element of $A_{0}$. An appeal to our Theorem 1 gives us a norm one projection $P_{\omega_{0}}$ based on a norming pair $\left(A_{\omega_{a}}, B_{\omega_{0}}\right), A_{\omega_{0}} \supset A_{\omega} W \supset B_{\omega_{\rho}} \supset B_{0}$, with $A_{\omega_{o}}|=| B_{\omega_{o}} \mid \stackrel{\omega_{o}}{=} \aleph_{0} A_{\omega_{o}}$ norm dense in $P_{\mathrm{w}_{o}}(X)$ and $B_{\omega_{o}}$ weak* dense in $P_{\omega_{o}}^{*}\left(X^{*}\right)$.

We shall proceed by transfinite induction to construct the other projections. Take $\omega_{o}<\alpha \leq \mu$ and, suppose we have defined for every ordinal $\beta$, $\omega_{o} \leq \beta<\alpha$, the projection $P_{\beta}$, based on a norming pair $\left(A_{\beta}, B_{\beta}\right)$, with

$$
A_{\beta} \supset\left\{x_{\alpha}: \alpha<\beta\right\}, B_{\beta} \subset W,\left|A_{\beta}\right| \leq|\beta| \text { and }\left|B_{\beta}\right| \leq|\beta|
$$

$A_{\beta}$ is norm dense subset of $P_{\beta}(X)$, and $B_{\beta}$ is weak* dense subset of $P_{\beta}^{*}\left(X^{*}\right)$, and $A_{\eta} \subset A_{\beta}, B_{\eta} \subset B_{\beta}$, if $\omega_{o} \leq \eta \leq \beta$ from where we have that

$$
P_{\eta} P_{\beta}=P_{\eta}=P_{\beta} P_{\eta} \text {, if } \omega_{o} \leq \eta \leq \beta \text {. }
$$

If $\alpha$ is not a limit ordinal, let $\gamma$ be the ordinal with $\gamma+l=\alpha$. Let $A_{\theta}$ be now equal to $A_{\gamma} \cup\left\{x_{r}\right\}$ and $B_{0}$ equal to $B_{r}$. 
Another application of Theorem 1 give us a projection $P_{\mathrm{a}}$ based on a norming pair $\left(A_{\mathrm{q}}, B_{\alpha}\right)$, with

$$
A_{\mathrm{a}} \supset A_{0}, B_{0} \subset B_{\mathrm{a}} \subset W \quad\left|A_{\mathrm{a}}\right| \leq \mid \alpha d \text { and }\left|B_{\mathrm{a}}\right| \leq|\alpha|
$$

$A_{\mathrm{a}}$ is norm dense subset of $P_{\mathrm{a}}(X)$, and $B_{\mathrm{a}}$ is weak* dense subset of $P_{\mathrm{a}}^{*}\left(X^{*}\right)$, from which we have that

$$
P_{\eta} P_{\mathrm{a}}=P_{\eta}=P_{\mathrm{a}} P_{\eta}, \text { if } \omega_{o} \leq \eta \leq \alpha
$$

If $\alpha$ is a limit ordinal, we write

and

$$
\begin{gathered}
A_{\mathrm{a}}=\cup\left\{A_{\beta}: \omega_{o} \leq \beta<\alpha\right\} \\
B_{\mathrm{a}}=\cup\left\{B_{\beta}: \omega_{o} \leq \beta<\alpha\right\} \subset W .
\end{gathered}
$$

It is clear that $\left(A_{\mathrm{a}}, B_{\mathrm{a}}\right)$ is a norming pair with $\varphi\left(B_{\mathrm{a}}\right) \subset A_{\mathrm{a}}$. Therefore the closed subspaces $\bar{A}_{\mathrm{a}}$ and $\bar{B}_{\mathrm{a}}$ verify the equivalent conditions of Proposition 5 , which means the existence of a norm-one projection $P_{\mathrm{a}}$ of $X$ onto $\bar{A}_{\mathrm{a}}$ along $B_{\mathrm{a}}^{\perp}$, in other words $P_{a}$ is based on the norming pair $\left(A_{a}, B_{a}\right)$. So we have

$$
\begin{gathered}
P_{\mathrm{a}}(X)=\overline{A_{\mathrm{a}}}=\overline{U\left\{P_{\mathrm{\beta}}(X): \omega_{\mathrm{o}} \leq \beta<\alpha\right\}} \\
\text { dens } \mathrm{P}_{\mathrm{a}}(X) \leq|\alpha|, \text { dens } \mathrm{P}_{\mathrm{a}}^{*}\left(X^{*}\right)_{\mathrm{a}} \leq|\alpha|,\left\|P_{\mathrm{a}}\right\|=1 \\
P_{\mathrm{\eta}} P_{\mathrm{a}}=P_{\mathrm{\eta}}=P_{\mathrm{a}} P_{\eta}, \omega_{\mathrm{o}} \leq \eta \leq \alpha
\end{gathered}
$$

Finally, $P_{\mu}$ is the identity operator and it is clear that

$$
\left\{P_{\mathrm{a}}: \omega_{\mathrm{o}} \leq \alpha<\mu\right\}
$$

is the P.R.I. we are looking for.

Q.E.D.

Corollary 4.1. (Vašák) Every weakly countably determined Banach space admits a P.R.I.

Corollary 4.2. (Fabian and Godefroy) Every dual Banach space with the Radon Nikodym property admits a P.R.I. 


\section{SHRINKING RESOLUTIONS OF IDENTITY}

We shall deal now with the study of Banach spaces $X$ where the projections can be simultaneously constructed for $X$ and $X^{*}$. For instance, where both $X$ and $X^{*}$ are W.C.G. [JZ1,3,5], or more generally $X$ and $X^{*}$ are W.C.D. [V]. Note that a Banach space with a W.C.D. dual is an Asplund space, indeed it has a projective generator on its dual. So we shall also deal with Asplund spaces which are W.C.D., [F2].

Recall the following definition:

Definition 6. A projectional resolution of identity

$$
\left\{P_{a}: \omega_{o} \leq \alpha \leq \mu\right\}
$$

on the Banach space $X$ is called a shrinking resolution of identity if the family of adjoint maps

$$
\left\{P_{a}^{*}: \omega_{o} \leq \alpha \leq \mu\right\}
$$

is also a P.R.I. on the dual Banach space $X^{*}$.

The P.R.I. construted above when $X^{*}$ has the Radon-Nikodym property is formed by projections which are not $\left(\omega^{*}-\omega^{*}\right)$ continuous in general. For instance, if $X=C\left(\left[0, \omega_{1}\right]\right) X^{*}$ has no P.R.I. consisting of $\left(\omega^{*}-\omega^{*}\right)$ continuous projections; indeed, the existence of such a resolution would imply the existence of a weak to weak continuous injection into $c_{0}(\Gamma)$ and this contradicts the fact that $\left[0, \omega_{1}\right]$ is not an Eberlein compact, [G2].

Note the following consequence of our construction:

Proposition 8. Let $X$ be an Asplund space and $\left\{P_{a} \div \omega_{0} \leq \alpha \leq \mu\right\}$ be the P.R.I. constructed in corollary 4.2. The following conditions are equivalent:

(i) Every $P_{\mathrm{a}}$ is weak* continuous.

(ii) $P_{\mathrm{a}}\left(X^{*}\right)$ is weak ${ }^{*}$ closed in $X^{*}$ for every $\alpha$.

(iii) If $P_{\mathrm{a}}$ is based on the norming pair $\left(A_{\mathrm{a}}, B_{\mathrm{a}}\right)$, then

$$
\bar{B}_{\mathrm{a}}^{\mathrm{du}}=\bar{B}_{\mathrm{a}}^{\alpha(\mathrm{x} \cdot \mathrm{x})}
$$

(iv) $\left\{P_{\alpha x}^{*} \cdot \omega_{0} \leq \alpha \leq \mu\right\}$ is a P.R.I. on $X$.

Proof. i $) \Rightarrow$ ii) $P_{\mathrm{a}}\left(X^{*}\right)$ is the orthogonal space $P_{a}^{*}(X)^{\perp}$ and therefore it is weak* closed. 
ii) $\Rightarrow$ iii) $P_{\mathrm{a}}\left(X^{*}\right)=\bar{B}_{\mathrm{a}}^{\| !}$, which is weak* closed, therefore $\bar{B}_{\mathrm{a}}^{\text {n॥ }}=\bar{B}_{\mathrm{a}}^{\text {od } x, n}$

iii) $\Rightarrow$ (iv) For every norming pair $\left(A_{\alpha} B_{\alpha}\right)$ we have

$$
A_{\mathrm{a}}^{\perp} \cap \bar{B}_{\mathrm{a}}^{\alpha x \times x)}=\{0\}
$$

and Proposition 5 says there is a norm one projection from $X$ onto $A_{a}^{\text {ill }}$ along $B_{a}^{\perp}$. It is clear that this projection coincides with the restriction of $P_{a}^{*}$ on $X$ and we obviously have all the conditions of a P.R.I because for a limit ordinal $a$ we have the identity

$$
A_{\mathrm{a}}=\cup\left\{A_{\mathrm{g}}: \omega_{\mathrm{o}} \leq \beta<\alpha\right\}
$$

Q.D.E.

Nevertheless, a more interesting result would be to know if we could change the P.R.I. of $X^{*}$ in order to obtain weak* to weak* continuity. The natural situation is provided by a projective generator on $X$ too:

Theorem 6. Let $X$ be an Asplund space. If $X$ has a projective generator defined on $X^{*}$, then $X$ has a shrinking P.R.I.

Proof. Let $\varphi: X^{*} \rightarrow 2^{x}$ be a projective generator on $X$, and $\psi: X \rightarrow 2^{x *}$ be the projective generator of $X^{*}$ as the dual of an Asplund space. If we proceed as we have done in Theorem 5, but using Theorem 4 instead of Theorem 1 to construct the projections, we obtain a projectional resolution of identity $\left\{P_{\mathrm{a}}: \omega_{0} \leq \alpha \leq \mu\right\}$ on $\mathrm{X}$, with every $P_{\mathrm{a}}$ based on the norming pair $\left(A_{\alpha}, B_{\mathrm{a}}\right)$ with all the properties of Theorem 5 , and such that $P_{a}^{*}$ is based on the norming pair $\left(B_{\alpha}, A_{\alpha}\right)$ for the projective generator $\psi$. It is clear now that $\left\{P_{a}^{*}: \omega_{o} \leq \alpha \leq \mu\right\}$ is a P.R.I. on $X^{*}$.

Q.E.D.

We include the following result due to Fabian [F2]

Corollary 6.. Let $X$ be a W.C.D. Banach space which is also Asplund. Then $X$ admits a shrinking P.R.I. and it is W.C.G.

Proof. We know that $X$ verifies the conditions of Theorem 6, and so $X$ has a shrining P.R.I. The fact that $X$ is also W.C.G. can now be obtained by an induction argument on the density character of $X$. Indeed, separable spaces are W.C.G. Let us suppose the result is true for every space with density character strictly less than the density character of $X$. Let $\left\{P_{\mathrm{a}}: \omega_{0} \leq \alpha<\mu\right\}$ be the shrinking P.R.I. constructed above. For every $\alpha$, the space $P_{\mathrm{a}}(X)$ is W.C.D. and Asplund with dens $\left(P_{\mathrm{a}}(X)\right) \leq|a|<\operatorname{dens}(X)=\mu$. Therefore $P_{\mathrm{a}}(X)$ is a W.C.G. Banach space. The result of Amir and Lindenstrauss give us a weak*-weak continuous linear injection $[\mathrm{AL}]$ 


$$
T_{\mathrm{a}}: P_{\mathrm{a}}^{*}\left(X^{*}\right) \rightarrow \mathcal{C}_{\mathrm{b}}\left(\Gamma_{\mathrm{a}}\right),\left\|T_{\mathrm{a}}\right\| \leq 1
$$

It is straightforward now to construct an injective, weak*-weak continuous linear injection $T: X^{*} \rightarrow \mathcal{C}_{0}(\Gamma)$ because $\left\{P_{a}^{*}: \omega_{a} \leq \alpha<\mu\right\}$ is a P.R.I. on $X^{*}$. Therefore, $B\left(X^{*}\right),\left[\sigma\left(X^{*}, X\right)\right]$ is linearly-homeomorphic to a weakly compact subset of the Banach space $c_{0}(\Gamma)$. A result of Lindenstrauss says that $X$ must be then W.C.G., [R].

Q.E.D.

A topological interpretation of the last corollary is the following (see [N] for definitions) result due to Alster [A].

Corollary 6.2. Let $K$ be a Hausdorff compact space. Then if $K$ is scattered and Gul'ko compact then it is an Eberlein compact space.

Proof. $C(K)$ is Asplund and W.C.D. Banach space, and so $C(K)$ is W.C.G. Thus $K$ is an Eberlein compact, [AL].

Q.E.D.

Related resuls can be found in the survey of Negrepontis [N], and the paper of Valdivia [V4].

\section{References}

[A] K. ALSTER. Some remarks on Eberlein Compacts, Fund. Math. 104 (1979) 43-46.

[AL] D. AMIR-J. LINDENSTRAuSs. The structure of Weakly Compact sets in Banach spaces, Ann. of Math. 88 (1968) 35-46.

[D1] J. DIESTEL. Geometry of Banach spaces. Selected topics, L.N.M. 485 Springer Verlag, Berlin (1975)

[D2] J. DIESTEL. Sequences and series in Banach spaces, G.T.M Springer Verlag, Berlin (1985)

[F1] M. FABIAN. On projectional resolution of identity on the duals of certain Banach spaces, Bull. Aust. Math. Soc. 36 (1987) 363-371.

[F2] M. FABIAN. Each weakly countably determined Asplund space admits a Frechet differentiable norm, Bull. Aust. Math.Soc. 36 (1987) 367-374.

[FG] M. FABIAN-G. GODEFROY. The dual of every Asplund space admits a projective resolution of identity. Studia Math. 91 (1988) 141-151.

[G1] G.GODEFroY. Boundaries of a convex set and interpolation sets, Math. Annalen 27 (1987) 173-184. 
[G2] G. Godefroy. Five lectures in geometry of Banach spaces, Seminar on Functional Analysis, 1987, edited by J. Orihuela and A. Pallares, University of Murcia.

[G] S. GUL'KO. The structure of spaces of continuous functions and their hereditary paracompactness, Russian Math. Surv. 34 (1979), 36-44.

[JR] J.E. JAYNE-C.A. ROGERS. Borel selectors for upper semicontinuous set valued maps, Acta Math. 155 (1985) 41-79

[JZ1] K. JOHN-V. ZIZLER. Projections in dual weakly compactly generated Banach spaces, Studia Math. 49 (1973) 41-50.

[JZ2] K. JoHN-V. ZIZLER. A note on renorming of dual spaces, Bull. Acad. Pol. Sci. Math. 21 (1973), 47-50.

[JZ3] K. JOHN-V. ZIZLER. Smoothness and its equivalents in weakly compactly generated Banach spaces, J. Funct. Anal. 15 (1974) 161-166.

[JZ4] K. JOHN-V. ZIZLER. Duals of Banach spaces which admits non trivial smooth functions, Bull. Aust. Math. Soc. 11 (1974) 161-166.

[JZ5] K. JOHN-V. ZIZLER. Weak compact generating in duality. Studia Math. 55 (1976) 1-20.

[L] J. LiNDENSTAUSS, On non separable reflexive Banach spaces, Bull. Amer. Math. Soc. 72 (1966), 967-970.

[M] S. MERCOURAKIS. On weakly countably generated Banach spaces, Trans. Amer. Math. Soc. 300 (1987) 307-327.

[NW] I. NAMIOKA-R.F. WHEELER. Gul'ko's proof of the Amir Lindenstrauss theorem, Contemp. Math. A.M.S. Vol. 52 (1986) 113-120.

[N] S. NEGREPONTIS. Banach spaces and topology, Handbook of set theoretic topology, Elsevier Sc. Pub. Edited by Kunen-Vaughan, 23 (1984) 1045-1142.

[O] J. ORIHUELA. Pointwise compactness in spaces of continuous functions, J. London Math. Soc. (2) 36 (1987) 143-152.

[P] R. POL. On pointwise and weak topology in function spaces, Warsaw University 4/84 (1984).

[R] H.P. ROSENTHAL. The hereditary problem for weakly compactly generated Banach spaces. Compositio Math. 28 (1974) 83-111.

[S] I. Singer. Bases in Banach spaces II. Springer Verlag. Berlín 1981.

[S1] C. STEGall. The Radon Nikodym property in conjugate Banach spaces, Trans. Amer. Math. Soc. 206 (1975) 213-223.

[S2] C. STEGall. The topology of certain spaces of measures. To appear in Seminar on Functional Analisis 1988, Universidad de Murcia.

[T] D.G. TACON. The conjugate of a smooth Banach space, Bull. Austr. Math. Soc. 2 (1970) 415-425.

[TA] M. TALAGRAND. Espaces de Banach faiblement K-analytiques. Ann. Math. 110 (1979) 407-438.

[V1] M. Valdivia. Espacios de Frechet de generación débilmente compacta. Coll. Mat. 38 (1987) 17-25.

[V2] M. VALDIVIA. Resolutions of identity in $C(K)$ spaces. To appear in Arch. der Math. 
[V3] M. VALDIVIA. Resolutions of identity in some metrizable locally convex spaces. Preprint.

[V4] M. VALDIVIA. Resolutions of identity in certain Banach spaces. Collec. Mat. 39 (1988) 124-140.

[V] L. VASÁK. On one generalization of weakly compactly generated Banach spaces, Studia Math. 70 (1980) 11-19.

Dept. Matemáticas

Universidad de Murcia

Sto. Cristo 1

30001 Murcia (ESPAÑA)
Dept. Análisis Matemático

Universidad de Valencia

Dr. Moliner 50

46100 Burjasot, VALENCIA

(ESPAÑA) 